Received: 2014.03.07 Accepted: 2015.04.13 Published: 2015.07 .27

\section{Primary Malignant Melanoma of the Esophagus: A Case Report and Review of the Literature}

Authors' Contribution:

Study Design A

Data Collection B Statistical Analysis C

Data Interpretation D Manuscript Preparation E

Literature Search F

Funds Collection G
ABEF 1 Antonio Navarro-Ballester

ABD 1 Susana De Lázaro-De Molina

BDE 2 John Gaona-Morales
1 Department of Diagnostic and Therapeutic Radiology, Hospital General Universitario de Castellón, Castellón de la Plana, Spain

2 Department of Pathology, Hospital General Universitario de Castellón, Castellón de la Plana, Spain
Corresponding Author: Conflict of interest:
Antonio Navarro Ballester, e-mail: antonio.navarroball@gmail.com

None declared

\begin{tabular}{|c|c|}
\hline Patient: & Male, 67 \\
\hline Final Diagnosis: & Primary malignant melanoma of esophagus \\
\hline Symptoms: & Dysphagia \\
\hline Medication: & - \\
\hline Clinical Procedure: & Endoscopic biopsy \\
\hline Specialty: & Gastroenterology and Hepatology \\
\hline Objective: & Challenging differential diagnosis \\
\hline Background: & $\begin{array}{l}\text { Primary malignant melanoma of the esophagus (PMME) is a rare cancer with a poor prognosis. It is often dif- } \\
\text { ficult to differentiate from non-epithelial malignant tumors, and immunohistochemical staining may be need- } \\
\text { ed to diagnose the condition. The mainstay of treatment is usually surgical with curative or palliative intent, } \\
\text { since radio- and chemotherapy do not really improve the outcome. The average survival rate after surgery is } \\
34.5 \text { months. At the time of diagnosis, } 40-80 \% \text { of cases have local regional lymph node metastases. }\end{array}$ \\
\hline Case Report: & 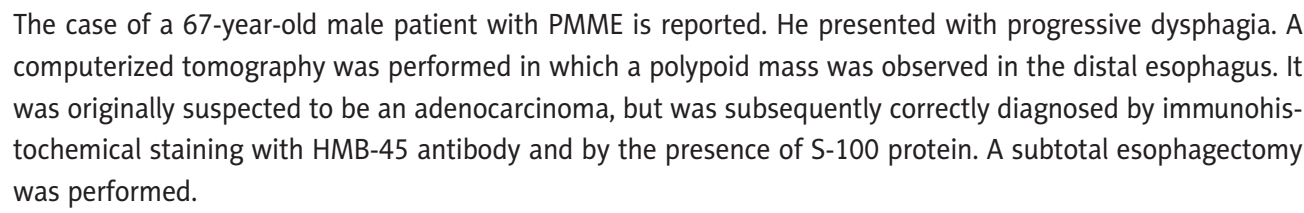 \\
\hline Conclusions: & $\begin{array}{l}\text { Very few cases of PMME have been reported in the literature and there is only limited clinical experience with } \\
\text { this disease. Therefore, it is very difficult to establish clear criteria for clinical recognition of this type of mela- } \\
\text { noma. Early histopathological confirmation of the character is essential for further treatment in case of confir- } \\
\text { mation of malignancy. }\end{array}$ \\
\hline
\end{tabular}

MeSH Keywords:

Full-text PDF: http://www.amjcaserep.com/abstract/index/idArt/894041

\section{Esophageal Neoplasms • Esophagectomy • Immunohistochemistry • Melanoma • Prognosis •} Tomography, Spiral Computed

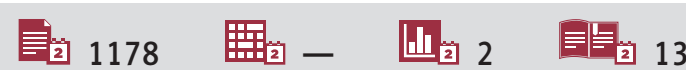




\section{Background}

Primary malignant melanoma of the esophagus (PMME) is a neoplasm that accounts for $0.1-0.2 \%$ of all primary neoplasms of the esophagus and $0.5 \%$ of visceral melanomas [1]. Baur described the first MPME in 1906 [2] and it has since been described in 340 different reports [3].

The MPME most often affects patients between the sixth and seventh decade of life. There is a 2.02:1 male predominance [4].

The most common clinical presentation is the onset of progressive dysphagia, chest pain, dyspnea, and weight loss [4]. Endoscopy shows MPME as one or more pigmented polypoid lesions, located in the middle and lower third of the esophagus [5].

Traditionally, the use of Computed Tomography (CT) with contrast has been suggested for the study of extension of PMME. However, recent studies recommend the 18 F-fluorodeoxyglucose-PET/CT to be used as a first-line modality for staging this tumor [6].

The MPME is usually very aggressive, showing nodal metastases at time of diagnosis in $40-80 \%$ of cases [7].
Due to its low frequency, MPME treatment is not standardized and it still remains a highly aggressive tumor. This case report presents a 67-year-old male with MPME originally suspected to be an adenocarcinoma by $\mathrm{CT}$, but subsequently correctly diagnosed by $\mathrm{HMB}-45$ and $\mathrm{S}-100$ protein immunohistochemistry. It includes a short review of the literature. The patient underwent surgical treatment and, till now, he is still alive for 6 months without any chemotherapy, radiotherapy and immunomodulatory therapy.

\section{Case Report}

A 67 year old man presented with a two months history of dysphagia for solids. He denied vomit or constitutional syndrome. A chest x-ray was done and was normal.

Subsequently, a Computed tomography (CT) of thorax revealed an intra-luminal mass of $35 \times 20 \mathrm{~mm}$ in the lower of esophagus, which was well-defined. There was no apparent local invasion or mediastinal lymph nodes enlargement. It was originally suspected to be an esophageal adenocarcinoma (Figure 1A, 1B).

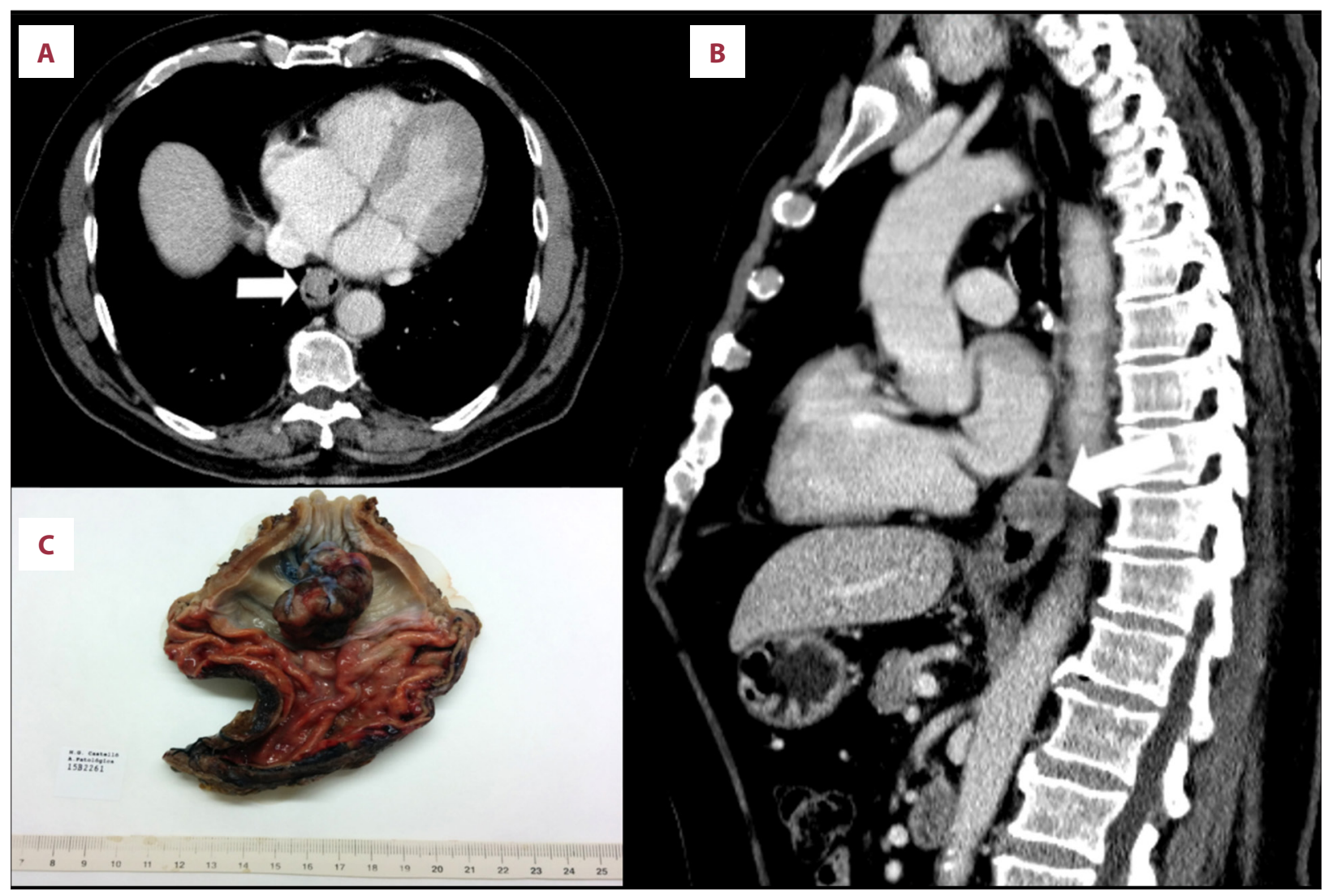

Figure 1. Axial (A) and coronal (B) CT showing a polypoid isodense mass in the esophagus, which expands and partially occupies the distal esophagus. (C) Surgical resection specimen showing a polypoid mass in the distal esophagus. 


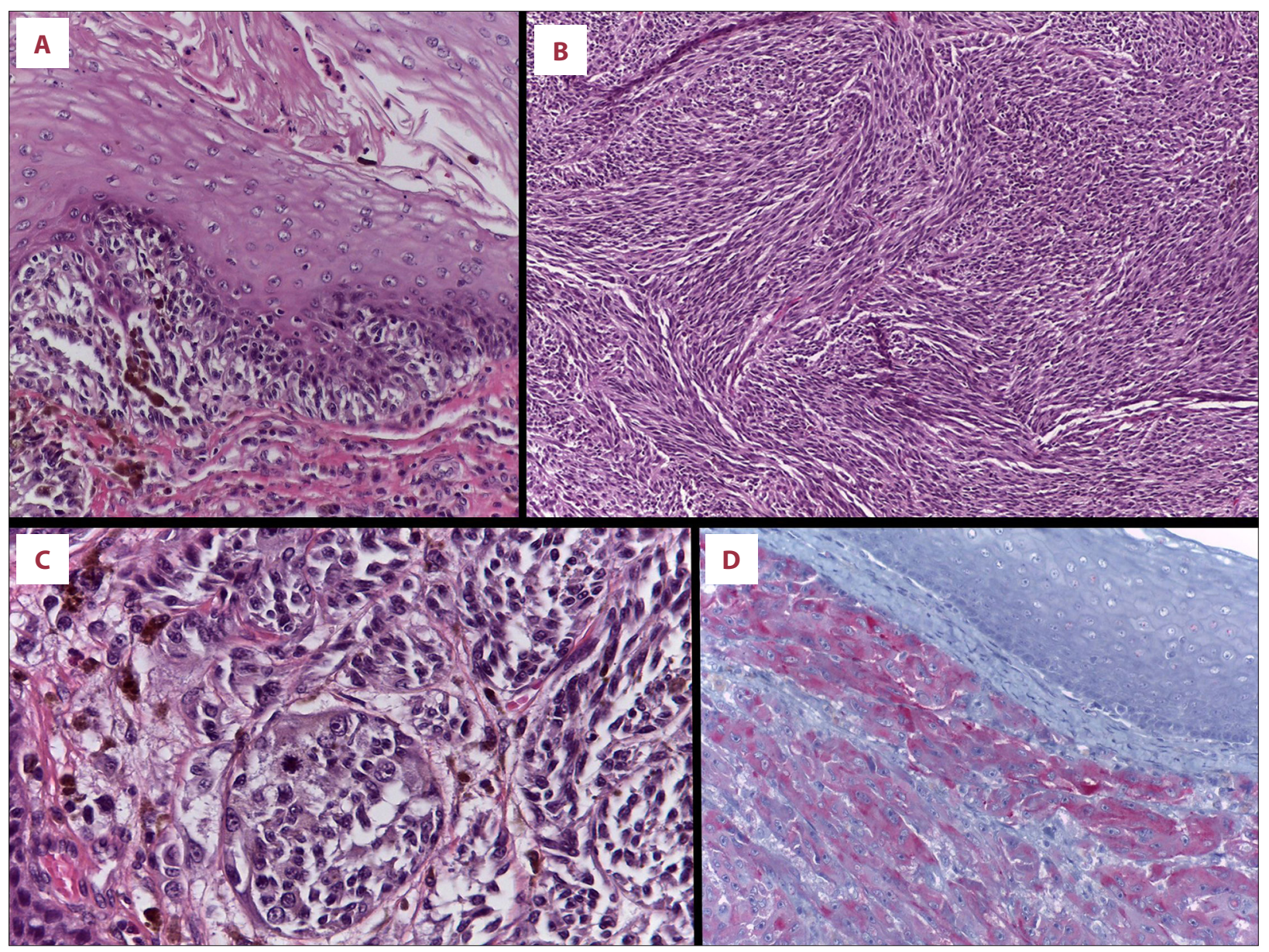

Figure 2. (A) Malignant melanoma with radial growth phase next to the infiltrating tumour (hematoxylin-eosin, $\times 200$ ). (B) Some tumor areas showing spindle-cell morphology (hematoxylin-eosin, $\times 100$ ). (C) Some areas showing epithelioid morphology, nuclear atypia, and mitotic activity (hematoxylin-eosin, $\times 200$ ). (D) The neoplastic cells were positive for Melan-A (immunohistochemistry, $\times 200$ ).

Given the findings, an endoscopy was performed. A pedunculated polypoid lesion of downy appearance was found $35 \mathrm{~cm}$ from the incisors. It was ulcerated and friable, occupying but not occluding the esophageal lumen.

An endoscopic biopsy of the lesion was performed. Samples were sent for pathology which revealed melanoma. No other findings of pathological significance were detected in the remainder of the study.

A positron emission tomography-computed tomography (PET$\mathrm{CT}$ ) was performed, being detected a hypermetabolism in the distal third of the esophagus (SUV 11.7). The rest of the examination did not show any evidence of other hypermetabolic lesions suggestive of a metastatic spread of the tumor.

With a preoperative staging IB (T2 N0 M0), a subtotal esophagectomy with double approach by laparoscopy and thoracoscopy and an Ivor Lewis intrathoracic anastomosis were performed.
Intraoperative findings were an exophytic polypoid tumor in the distal esophageal third, which did not invade the adventitia and apparently did not affect the muscle itself. It was also observed pigmented para-esophageal and subcarinal lymphadenopathies. These were excised and sent with the resected specimen of the esophagus to the pathology department to be examined.

The macroscopic examination of the surgical specimen showed a pigmented black, polypoid lesion measuring $4.3 \times 2.4 \times 2 \mathrm{~cm}$ (Figure 1C).

The microscopic examination also showed that the tumor was composed of polygonal and spindle-shaped cells in a sheetlike growth pattern with minimal inflammation and stromal reaction (Figures $2 \mathrm{~A}-2 \mathrm{C}$ ). Furthermore it showed some melanin pigment cells. Radial growth was seen in mucosa surrounding the tumor (Figure 2A). The tumor cells were stained positive with S100, Melan-A and HMB45 (Figure 2D). A metastasis was 
seen in one regional lymph node. The diagnosis was a primary malignant melanoma of the esophagus.

The patient had a good clinical outcome after surgery and was discharged home 14 days after surgery. He has subsequently undergone a CT and a PET-CT scans and remains disease free for over 6 months.

\section{Discussion}

The PMME is an extremely rare neoplasm [1] that manifests itself in most cases as a progressive dysphagia, just as it did in our case [4].

Currently, the diagnostic sequence is still not properly established although a CT with intravenous contrast is an option for an extension study. Our patient showed a nonspecific CT presentation of the primary lesion, as well as other authors have reported $[4,6]$. Therefore, the histological confirmation is always necessary to establish a definitive diagnosis of melanoma as Sahoo et al. suggested [8]. Other forms of useful images are MRI and CT-enterography, and most importantly, the PET-CT [7].

Sanchez et al. [5] found that primary malignant melanoma of the esophagus occurred mostly (>90\%) in the mid and lower third of esophagus.

The differential diagnosis includes spindle cell carcinoma, leiomyosarcoma and Kaposi's sarcoma. It is difficult to differentiate these MPME esophageal tumors based on radiological criteria but there are some manifestations that may be more suggestive of some of them [7]. For example, in the esophagogram, the spindle cell carcinoma is seen as a large intraluminal lobed mass that expands the light without causing obstruction. In the CT, the leiomyosarcoma shows as a heterogeneous exophytic mass with areas of necrosis, while Kaposi's sarcoma shows as multiple submucosal masses or polypoid lesions [7].

Other malignancies such as adenocarcinoma of the esophagus or the squamous cell carcinoma of the esophagus also may rarely manifest as a polypoid mass. In these neoplasms it is more common to see an eccentric or circumferential wall thickening greater than $5 \mathrm{~mm}$.

\section{References:}

1. Volpin E, Sauvanet A, Couvelard A, Belghiti J: Primary malignant melanoma of the esophagus: a case report and review of the literature. Dis Esophagus, 2002; 15: 244-49

2. Iwanuma Y, Tomita N, Amano T et al: Current status of primary malignant melanoma of the esophagus: clinical features, pathology, management and prognosis. J Gastroenterol, 2012; 47: 21-28
Histologically, melanomas exhibit considerable variability in cell size and shape, both from tumor to tumor and within a given tumor $[8,9]$. They can mimic other malignancies such as malignant lymphoma, small round cell sarcoma, spindle cell sarcoma, gastrointestinal stromal tumor, spindle cell carcinoma, or even epidermoid carcinoma [9]. In the event of difficulty in establishing the diagnosis, particularly in amelanotic cases, immunohistochemical examination is suggested to establish full diagnosis. These examinations use antibodies against HMB45 and S-100 protein [10].

Currently, MPME prognosis is poor, with an average survival rate of 34.5 months after surgery [11]. The most important prognostic factor is the presence of nodal metastases [11]. For this purpose, an FDG-PET may be useful; its sensitivity is directly proportional to the size of the tumor, as reported by Crippa et al. [12].

Kranzfelder et al. have reported disease control after administration of chemoradiotherapy but there are no definitive data about the effect of postoperative chemotherapy or radiotherapy on overall 5-year survival [13]. The only treatment option that has a real impact on the survival rate is surgical treatment, but other therapies can be used in palliative care [4].

\section{Conclusions}

PMME is a rare but often fatal disease. This case report presents a patient with PMME by positive results for HMB-45 and S100 protein, treated by surgical resection without chemotherapy. Six months after surgery, the patient is alive without tumor recurrence. The diagnosis of PMME should combine clinical symptoms, auxiliary examination, pathological examination, and immunohistochemistry. We propose that the effectiveness of diagnostic guidelines or treatment will rise with an increase in the number of PMME case reports.

\section{Statement}

We received no financial support for this study. We have no conflict of interest to declare.

3. Terada T: A clinicopathologic study of esophageal 860 benign and malignant lesions in 910 cases of consecutive esophageal biopsies. Int J Clin Exp Pathol, 2013; 6: 191-98

4. Yu Q Yuan S: Primary malignant melanoma of the esophagus with subtotal esophagectomy: a case report. Int J Clin Exp Med, 2014; 7: 4519-22

5. Sanchez AA, Wu TT, Prieto VG et al: Comparison of primary and metastatic malignant melanoma of the esophagus: clinicopathologic review of 10 cases. Arch Pathol Lab Med, 2008; 132: 1623-29 
6. Pfannenberg C, Schwenzer N: Whole-body staging of malignant melanoma: Advantages, limitations and current importance of PET-CT, whole-body MRI and PET-MRI. Radiologe, 2015; 55: 120-26

7. Lin $P$, Lee $R$, Chern $M$ et al: Primary malignant melanoma of the esophagus. J Chin Med Assoc, 2006; 69: 334-37

8. Sahoo MR, Gowda MS, Kaladagi RM: Primary amelanotic melanoma of the rectum mimicking adenocarcinoma. Am J Case Rep, 2013; 14: 280-83

9. Lagha A, Ayadi M, Krimi S et al: Primary anorectal melanoma: A case report with extended follow-up. Am J Case Rep, 2012; 13: 254-57
10. Dominiak M, Gerber H, Kubasiewicz-Ross P et al: Amelanotic malignant melanoma in the oral mucosa localization. Am J Case Rep, 2011; 12: 159-62

11. Harada K, Mine S, Yamada K et al: Long-term outcome of esophagectomy for primary malignant melanoma of the esophagus: a single-institute retrospective analysis. Dis Esophagus, 2015 [Epub ahead of print]

12. Crippa F, Leutner $M$, Belli $F$ et al: Which kinds of lymph node metastases can FDG PET detect? A clinical study in melanoma. J Nucl Med, 2000; 41 1491-94

13. Kranzfelder M, Seidl S, Dobritz M, Brücher BL: Amelanotic esophageal malignant melanoma: case report and short review of the literature. Case Rep Gastroenterol, 2008: 2(2): 224-31 